Jean LEVASSEUR, «La poésie humoristique : Rémi Tremblay et les relations amoureuses au XIX ${ }^{\mathrm{e}}$ siècle canadien ", dans La comédie de l'amour. Actes du colloque du CORHUM organisé en 2005 par Lucie Joubert, @ nalyses, printemps-été 2006

Jean LEVASSEUR

\title{
La poésie humoristique : Rémi Tremblay et les relations amoureuses au $\mathrm{XIX}^{\mathrm{e}}$ siècle canadien
}

Né en 1847 dans un petit village de la rive sud du Saint-Laurent, Rémi Tremblay ${ }^{1}$ vécut ses enfance et adolescence trimbalé entre les misères de la vie dans les terres agricoles québécoises et les manufactures de textile d'une Nouvelle-Angleterre alors en pleine expansion. Il ne put ainsi effectuer que de maigres études primaires, ce qui ne l'empêchera pas de se lancer férocement, lorsque, plus tard, le temps le lui permettra, dans l'étude de l'algèbre, des langues et de la versification. De retour au Canada en 1861, alors que planaient les rumeurs d'une guerre civile américaine, le jeune adolescent occupa pendant deux ans des emplois variés qui ne menaient à rien. En 1864, déçu et ennuyé par la vie, il mentit sur son âge - il n'avait alors que 16 ans - et s'engagea dans l'armée américaine, participant aux dix-huit derniers mois de ce sanglant conflit qui enleva la vie à plus de 600000 jeunes Américains, dont six dans l'une des pires prisons des États confédérés, la prison Libby (Richmond, Virginie).

Un concours de circonstances l'amena ensuite à devenir correspondant pour un journal franco-américain, Le Protecteur de SaintAlban (Vermont), puis pour Le Pionnier de Sherbrooke des Cantons de l'Est, au Québec, où il y emporta bientôt ses pénates. Vers la fin de la décennie, il se retrouva à Montréal où, dans les années 1880 et 1890, il dirigea certains des hebdomadaires les plus importants et influents de son époque, dont Le Courrier de Montréal et La Patrie. Il devint

\footnotetext{
${ }^{1}$ Les informations biographiques présentées ici proviennent de recherches de l'auteur, présentement en cours, et dont les objectifs sont la parution d'une anthologie de poésie commentée, puis d'une biographie de Rémi Tremblay. On peut d'ores et déjà trouver certaines de ces informations dans les publications de Jean Levasseur référencées dans la bibliographie.
} 
Jean LEVASSEUR, «La poésie humoristique : Rémi Tremblay et les relations amoureuses au XIX ${ }^{\mathrm{e}}$ siècle canadien ", dans La comédie de l'amour. Actes du colloque du CORHUM organisé en 2005 par Lucie Joubert, @ nalyses, printemps-été 2006

également, en 1884, le premier traducteur à la Chambre des communes d'Ottawa à obtenir son poste au terme d'épreuves linguistiques plutôt que grâce au sempiternel favoritisme politique. Entre ces multiples emplois, il publia deux œuvres théâtrales, un roman - Un revenant, écrit en partie autobiographique qui reprenait certains des épisodes vécus par lui durant la guerre de Sécession américaine -, ainsi que cinq recueils de poésies et de chansons.

\section{La poésie canadienne-française du $\mathrm{XIX}^{\mathrm{e}}$ siècle et la critique}

L'une des grandes tragédies de la poésie canadienne-française du XIX siècle fut peut-être de vouloir invariablement se comparer, tant chez ses auteurs que chez ses critiques, aux créations et aux évaluations de leurs contemporains de l'Hexagone. Loin d'être autonome, le champ littéraire canadien, pour reprendre la terminologie de Bourdieu, était alors fortement dépendant de l'institution française, tant dans sa critique que dans ses modes stylistiques. Conscient de cette situation, Joseph Doutre déplorait déjà, dans Les Fiancés de 1812 (1844), cet amour inconditionnel — et exagéré — de l'étranger, si inconditionnel qu'il rendait difficile, sinon impossible, la réussite canadienne :

Telle est la généralité de ce préjugé en faveur de l'étranger que, sur quarante mille hommes lettrés, on n'en trouvera pas dix qui ne soient possédés de fureur pour les productions européennes; et à peine en rencontrera-ton mille qui liront avec plaisir le travail d'un de leurs concitoyens, de quelque genre qu'il soit. (1844, p. 2)

Quarante ans plus tard, et dans un contexte légèrement différent, Rémi Tremblay se fera lui-même l'écho de la déception du célèbre membre de l'Institut canadien :

C'est juste, et j'admettrais qu'un vulgaire écrivain ${ }^{2}$ Se plaindrait bien à tort, s'il réclamait en vain

\footnotetext{
${ }^{2}$ Entendre ici « écrivain canadien ».
} 
Jean LEVASSEUR, «La poésie humoristique : Rémi Tremblay et les relations amoureuses au XIX ${ }^{\mathrm{e}}$ siècle canadien ", dans La comédie de l'amour. Actes du colloque du CORHUM organisé en 2005 par Lucie Joubert, @ nalyses, printemps-été 2006

Une part des profits de l'imprimeur-libraire.

Arbitre du bon goût, ce mortel n'a que faire

Des écrits du terroir, puisqu'il a sous la main

Des livres imprimés dans l'ancien hémisphère [...]. («In forma pauperis », 1888b, p. 77)

Certes, les poètes canadiens avaient attiré ce «malheur» sur eux, leur inspiration formelle provenant inconditionnellement, durant tout le siècle, d'abord des représentants du classicisme français, particulièrement Boileau et Molière, puis des romantiques, avec en tête Lamartine, Hugo, Musset et Chateaubriand, et ce, jusqu'à l'École littéraire de Montréal qui se laissera, elle, guider par les muses du Parnasse, du symbolisme et des poètes décadents.

En regard de ces canons argumentaires favorisés par la critique du XIX $^{\mathrm{e}}$ siècle, qui renvoie toujours vers les grands modèles de l'Hexagone, la poésie de Rémi Tremblay ne figure certes pas au panthéon des mythes et des légendes. De 1879, année où il fit paraître, sous le pseudonyme de "Père Louison", son grinçant Chansonnier politique du Canard, à 1912, où il présenta aux lecteurs le plus classique, et le moins intéressant, de ses volumes en vers, Vers l'idéal, cinq recueils de poèmes et chansons virent le jour. Quelques critiques sauront reconnaitre les qualités spirituelles de ses compositions: "C'est un bon placement pour ceux qui aiment à se désopiler la rate » (1883a), s'exclamera simplement un journaliste du Progrès de l'Est, Rémi Tremblay «excelle dans le genre satirique»(1883b), commentera Pierre Mignault dans la Revue canadienne; Louis Fréchette louangera son "vers plein, robuste, ingambe», et soulignera son "trait sanglant», véritable « coup de fouet à la Juvénal » (1888a). La plupart d'entre eux toutefois, tributaires des critères d'évaluation alors en vogue, déploreront son choix stylistique. Il devrait prendre "son rôle de poète un peu plus au sérieux » (1883b), ajoutera Mignault; après avoir cité Ion, de Platon, le père de la critique canadienne, Mgr Camille Roy, dira de lui qu'«il a de l'originalité parfois dans ses conceptions, du pittoresque dans son regard, de l'élan dans sa verve ", mais l'ensemble de sa poésie nécessiterait selon lui un resserrement et une intensification de sa pensée, complétera-t-il (1923a). Inspiré par ces 
Jean LEVASSEUR, «La poésie humoristique : Rémi Tremblay et les relations amoureuses au XIX ${ }^{\mathrm{e}}$ siècle canadien ", dans La comédie de l'amour. Actes du colloque du CORHUM organisé en 2005 par Lucie Joubert, @ nalyses, printemps-été 2006

mêmes références institutionnelles, le professeur et essayiste Guy Frégault affirmera, un demi-siècle plus tard, que Tremblay n'avait « rien apporté de neuf à notre littérature» (1996, p. 76), une conclusion sans doute un peu trop hâtive qui plongera cet auteur dans l'oubli jusqu'à la parution d'une édition critique (2003) de son roman, Un revenant $3^{3}$.

Il serait en effet injuste de terminer là l'évaluation. En effet, les critères d'appréciation modernes font trop souvent fi d'un élément indispensable au bon jugement de la qualité d'un écrivain : la réception populaire. Si sa présence n'est pas essentielle, loin de là, à la reconnaissance de l'œuvre de génie, le refus d'en tenir compte tient presque de la mauvaise volonté et de la critique bâclée, alors qu'elle peut involontairement reléguer aux oubliettes des participants importants à la petite histoire d'un peuple. Malheureusement, le manque d'intérêt pour cette histoire et le sentiment de supériorité de la société contemporaine ont rapidement éliminé le $\mathrm{XIX}^{\mathrm{e}}$ siècle canadien, à quelques rares exceptions près, et les œuvres les plus méritoires n'ont pas toujours été reconnues. Cet élément fondateur de la littérature, ainsi que son omission, ont ainsi effacé de nos mémoires bien des artistes de valeur, dont Rémi Tremblay.

\footnotetext{
${ }^{3}$ Dans la seconde moitié du $\mathrm{XX}^{\mathrm{e}}$ siècle, Tremblay ne fera l'objet, partiel, que d'une seule thèse de doctorat (voir 1968), qui portait sur la poésie francoaméricaine. La thèse fut publiée quelques mois plus tard aux Éditions de l'Abbaye de Thélème à l'Étang des Lys (Somerworth, New Hampshire). La recherche dans les archives, dans les années 60, était beaucoup plus complexe qu'aujourd'hui, et le jeune chercheur fit preuve d'une sérieuse méconnaissance du XIX ${ }^{\mathrm{e}}$ siècle, multipliant les erreurs et les interprétations faciles, mais fausses. Il semble également avoir été incapable de séparer le «je» narrateur des poésies de Tremblay et l'auteur lui-même, ce qui l'amène à tirer des conclusions souvent risibles sur la vie et la personnalité de cet écrivain. M. Chassé répéta ces mêmes maladresses dans le Dictionnaire des cuvres littéraires du Québec, alors que l'on fit appel à lui pour toutes les entrées sur Tremblay (hormis celle de son premier recueil). L'on doit par ailleurs à Suzanne Lafrenière, professeure à l'Université d'Ottawa, le grand mérite d'avoir sauvé cet écrivain de l'oubli total par la préservation des archives de Jules Tremblay, fils de Rémi, à qui elle consacra plusieurs fructueuses années de recherches.
} 
Jean LEVASSEUR, «La poésie humoristique : Rémi Tremblay et les relations amoureuses au XIX ${ }^{\mathrm{e}}$ siècle canadien ", dans La comédie de l'amour. Actes du colloque du CORHUM organisé en 2005 par Lucie Joubert, @ nalyses, printemps-été 2006

Tremblay était en effet beaucoup plus à l'aise dans la chronique du quotidien que dans les énoncés de poésie de facture lyrique, que l'on considère aujourd'hui encore comme «supérieure », plutôt que simplement "différente», comme en témoignent pourtant les reconnues et respectées théories de l'altérité et de l'exiguité, dans un autre secteur des études littéraires. Grinçants témoins de la politique fédérale, provinciale et municipale de son époque, les chansons satiriques et humoristiques qu'il composa, et qui comptent pour plus de $50 \%$ de ses quatre premiers recueils, complètent avec saveur la petite histoire du pays et rendent indélébiles ses scandales, sa corruption et ses maquignonnages. Observateur fin et chevronné, Tremblay permit à sa plume féconde de rédiger une vaste chronique de la vie des gens ordinaires, de ses tics, de ses coutumes et de ses doux travers, en cela annonciateur des Mary Travers — La Bolduc et Émile Coderre - Jean Narrache - , deux personnalités importantes de la culture populaire québécoise de la première moitié $\mathrm{du} \mathrm{XX}^{\mathrm{e}}$ siècle. Tous ces commentaires sociaux, il les transposait dans une poésie dénonciatrice qui empruntait à l'une ou l'autre de deux tendances: une voix sérieuse, où il s'inspirait évidemment des structures de la poésie traditionnelle, et une voix humoristique, dominante, où il laissait aller librement son imagination, ce qui lui permettait de jouer, dans un vocabulaire populaire, riche et attachant, sur des sujets très souvent inédits.

\section{Le statut des chansonniers au $\mathrm{XIX}^{\mathrm{e}}$ siècle}

Il est difficile pour le lecteur contemporain de remettre en son contexte véritable le rôle exact de la chanson dans la société du XIX siècle; mais, et pour reprendre les mots des folkloristes Maurice Carrier et Monique Vachon, «il n'est pas de chronique sociale, économique et politique plus sûre que la chanson» (1977, p. 9). Tirades de dénonciations et de scandales, refrains politiques, satire d'événements et de personnages, la chanson était partout et jouissait d'une popularité indubitable, allègrement illustrée par ses innombrables présences dans les journaux de l'époque et les réactions, souvent violentes, que plusieurs d'entre elles provoquèrent. Certains compositeurs devinrent même des légendes vivantes, ou du moins des 
Jean LEVASSEUR, «La poésie humoristique : Rémi Tremblay et les relations amoureuses au XIX ${ }^{\mathrm{e}}$ siècle canadien ", dans La comédie de l'amour. Actes du colloque du CORHUM organisé en 2005 par Lucie Joubert, @ nalyses, printemps-été 2006

personnalités marquantes de leur époque. Louis Fréchette témoigne de ce fait dans ses Originaux et détraqués, alors qu'il consacre un chapitre entier au chansonnier populaire d'origine incertaine Grosperrin, qui se plaisait lui-même à se définir comme un " philosophe cosmopolite » et un «enfant de l'humanité » (1892, p. 168). À son tour, il saura inspirer un jeune Rémi Tremblay, tout frais revenu de la guerre de Sécession américaine, qui s'en souviendra comme d'un « incomparable trouvère » (1923b, p. 89).

Une quinzaine d'années plus tard, lorsqu'Hector Berthelot lui demandera de collaborer par une chanson satirique hebdomadaire à son journal irrévérencieux, Le Canard, Tremblay se souviendra de son ton léger et grinçant ainsi que de sa qualité d'observation du quotidien. Grosperrin ne sera toutefois pas la seule source d'inspiration du jeune journaliste récemment arrivé dans le giron de la métropole canadienne. Ses écrits témoignent en effet de l'influence certaine de plusieurs autres chansonniers français, dont les incomparables Pierre-Jean Béranger et Gustave Nadaud, qu'il cite d'ailleurs occasionnellement. Tremblay sut également utiliser au meilleur de ses possibilités la chanson de type «mazarinade » du XVII ${ }^{\mathrm{e}}$ siècle, dont il soutira le venin et la satire acerbe, et l'employa comme à l'époque, c'est-à-dire comme la seule opposition possible au pouvoir en place, particulièrement ici en faveur de l'ouvrier, du petit peuple et du laissépour-compte. Qui plus est, ce grand défenseur qu'il était de la mère patrie, du travailleur et des principes politiques et sociaux de la nouvelle république française n'aura pu qu'être inspiré par la nouvelle vague de chansons mises au service des défenseurs de la philosophie républicaine (Ca ira, La Carmagnole, La Marseillaise, etc.), hommes et femmes qui se retrouveront dans ces petites boîtes parisiennes où s'était réfugié l'esprit révolutionnaire et où l'on applaudissait des chansonniers ouvriers (voir 1990), dont certains sont arrivés jusqu’à nous: Henri Avenel, Vinçart, J.-B. Clément, Charles Gille, Eugène Pottier, etc.

\section{Le couple et la poésie d'inspiration féminine}

Ainsi interprétés, les écrits de Rémi Tremblay se transforment en une poésie aussi amusante que succulente, une chronique de fin de siècle 
Jean LEVASSEUR, «La poésie humoristique : Rémi Tremblay et les relations amoureuses au XIX ${ }^{\mathrm{e}}$ siècle canadien ", dans La comédie de l'amour. Actes du colloque du CORHUM organisé en 2005 par Lucie Joubert, @ nalyses, printemps-été 2006

où le lecteur se voit ouvrir le chemin vers les couleurs, les émotions et les sensations de toute une époque, de toute une société, une atmosphère qui, autrement, aurait à jamais été perdue. Dans cette vaste chronique populaire, Tremblay se plut à mettre en scène le couple et ses amusantes ou moins amusantes difficultés domestiques.

Deux types de femmes se démarquent dans la poésie chansonnière de Tremblay: la femme privée, dominante en nombre d'écrits, et la femme publique, plus discrète tant dans la réalité que dans ses fictions. La première se définit, dans une évidente recherche du sourire inhérent à la chanson du quotidien, par son opposition à son élément le plus naturel: l'homme. Il est ainsi possible de suivre toute l'évolution des relations intimes entre les deux êtres, depuis les premières fréquentations jusqu'au mariage et jusqu'à la découverte de cette femme «nouvelle», que l'homme «dénude » dans son intimité d'abord, puis dans sa prise de conscience de l'existence d'un univers domestique résolument matriarcal. La seconde, présente dans une poignée de créations, témoigne du féminisme grandissant et du désir du prétendu sexe faible de participer aux activités publiques et politiques de la société. Tremblay y joue alors du carnavalesque, se plaisant à inverser les rôles et imaginer les femmes dans divers rôles traditionnellement masculins, pendant que l'homme, passif, assiste de la maison aux inévitables renversements sociaux :

La femme n'aurait plus d'enfant,

Mais elle porterait culotte.

En pantalon pied d'éléphant, Elle deviendrait matelote.

Sous le harnais d'un cuirassier, On la verrait aux hippodromes;

Les corsets seraient en acier

Si les femmes étaient des hommes. (1912)

La femme privée, quant à elle, joue de tous les registres, de la matrone à la femme sensuelle, une aberration dans un Québec ultraconservateur, dominé par un clergé fermé et intransigeant, de la commère à la mégère, qui encourage son fils à épouser « la fill' du gros 
Jean LEVASSEUR, «La poésie humoristique : Rémi Tremblay et les relations amoureuses au XIX ${ }^{\mathrm{e}}$ siècle canadien ", dans La comédie de l'amour. Actes du colloque du CORHUM organisé en 2005 par Lucie Joubert, @ nalyses, printemps-été 2006

notaire » («La fill' d'ma bell'-mère », 1883c, p. 115) et dont la notoriété et l'influence sont telles qu'elle fait concurrence aux journaux (voir «Le commérage », 1883c, p. 100). Dans la création de ses effets humoristiques, Tremblay mise avant tout sur l'emploi d'un humour axé sur le quotidien et sur son dérivé, l'identification, mais une identification au deuxième degré seulement, laquelle permet de rire de la souffrance du prétendant ou du marié. Son personnage comique ressemble ainsi souvent au clown triste que rendra célèbre Charlie Chaplin, quelques décennies plus tard: insouciant, bon vivant, merveilleusement docile, ici face au pouvoir de la femme dans son espace privé, et sachant s'adapter à tous les échecs et à tous les aléas de la vie, il se démarque par une incroyable naïveté qui le rend sympathique aux yeux de tous. C'est à travers ses yeux que l'on explore, la plupart du temps, cette femme qui émergera de la fin du $\mathrm{XIX}^{\mathrm{e}}$ siècle.

Le premier rapport significatif homme/femme demeure évidemment celui des fréquentations; nulle chanson de Tremblay ne témoigne avec plus de finesse et d'humour des étapes traditionnelles de la cour et de la séduction que «La Fille à Baptiste », une chanson prométhéenne sur les efforts de l'homme pour conquérir sa bien-aimée, efforts qui l'amènent à devenir un habitué de la buvette de son père, où elle travaille quotidiennement, jusqu'à ce qu'elle lui envoie, au terme de longues fréquentations d'affaires :

S'rait-ce mon amour ou l'whisky qui m'brûle?

Si ça n's'arrêt' pas bientôt j'creverai!

Ell' m'a dit l'aut' jour : « Ça c'est ridicule, Quand vous n'boirez plus j'vous épouserai.» (1883c, p. 112)

Certes, il est de ces fréquentations permettant des rencontres plus intimes, qui peuvent aller jusqu'au baiser et aux caresses; mais ce plaisir n'est pas sans sa contrepartie de risques, personnifiée généralement par l'apparition soudaine d'un chaperon, ou d'un parent, incontournable étape d'une éducation masculine d'un tout autre type : 
Jean LEVASSEUR, «La poésie humoristique : Rémi Tremblay et les relations amoureuses au XIX ${ }^{\mathrm{e}}$ siècle canadien ", dans La comédie de l'amour. Actes du colloque du CORHUM organisé en 2005 par Lucie Joubert, @ nalyses, printemps-été 2006

J'allongeais déjà la babine

Pour lui fair' péter l'bec, Lorsque je reçus sur l'échine

Un coup d'pied pas mal sec.

C'était l'papa, d'humeur maussade, Qui v'nait mal à propos

Gâter l'plaisir d'notre embrassade

En tapant sur mon dos.

$[\cdots]$

Avant d'y r'tourner, j'me frai faire

Un fond d'culott' d'airain :

Faut, en amour comme à la guerre,

Protéger l'arrièr' train. ("Fâcheux dénouement », 1883c, p. 39-40)

Comme la première flamme n'est pas toujours la femme de sa vie, l'homme découvre bientôt qu'il est bien difficile de trouver la partenaire idéale, mais, à force d'efforts et de persévérance, la réussite l'attend un jour, quelquefois, cependant, grâce au dépit de celle pour qui monsieur n'est que le deuxième choix. Heureusement, le mariage, institution incontournable, finit toujours par avoir le dessus sur le triste célibat. Une fois donc l'épouse sélectionnée, et la noce célébrée, non sans difficultés et mémorables anecdotes, le nouveau marié, être toujours aussi bonasse et un peu niais, explore les traits jusqu'alors insoupçonnés de son épouse. Insensiblement, il en vient ainsi, par exemple, à personnifier l'ambition de sa femme, mais ne remarque bien sûr rien de son caractère pour le moins autarcique, parce que

D'mon sort elle a tant d'souci,

Qu'ell' me houspill' sans merci. (" La fill' d'ma bell'mère », 1883c, p. 117) 
Jean LEVASSEUR, «La poésie humoristique : Rémi Tremblay et les relations amoureuses au XIX ${ }^{\mathrm{e}}$ siècle canadien ", dans La comédie de l'amour. Actes du colloque du CORHUM organisé en 2005 par Lucie Joubert, @ nalyses, printemps-été 2006

Et si elle n'est pas heureuse des résultats des efforts professionnels de son époux, en toute honnêteté, madame n'hésite pas à le lui dire :

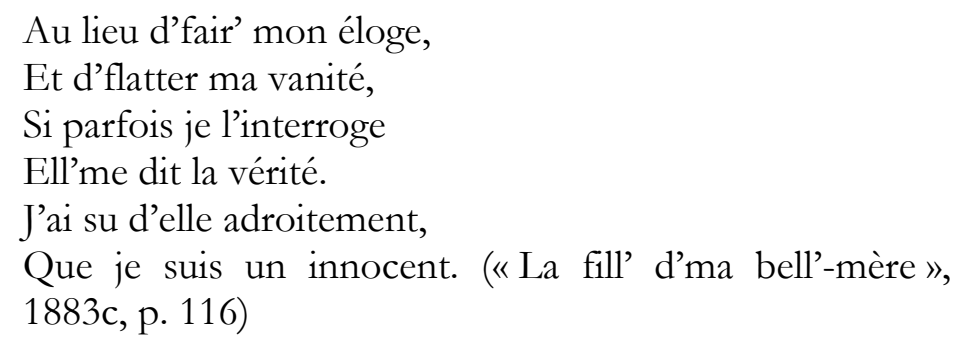

Qui plus est, la nouvelle épouse peut aussi être atteinte du véniel péché de jalousie; malheureusement, toutefois, le marié, victime d'un quotient intellectuel légèrement déficient, ou d'un quotient amoureux impressionnant, pressent cette expression féminine comme un simple désir de protection :

Quelquefois je vais en ville,

Mais je n'crains pas le danger,

Ma femme a quelqu'un qui m'file

Et qui saurait me protéger:

Elle connait ma candeur,

Et veut que je garde ma pudeur. («La fill' d'ma bell'mère », 1883c, p. 116)

Homme de son temps, Tremblay ne pouvait non plus ne pas remarquer l'intérêt particulier de la femme pour la pratique incessante de la parole et les commérages entre voisines, destructrices de réputations. Pour l'amoureuse des querelles, le résultat est toujours le même :

Revêche et bégueule

J'aime le combat :

En deux tours de gueule

Je clos le débat. («La mégère », 1883c, p. 126)

Cet ensemble de traits, qui démontre un univers domestique 
Jean LEVASSEUR, «La poésie humoristique : Rémi Tremblay et les relations amoureuses au XIX ${ }^{\mathrm{e}}$ siècle canadien ", dans La comédie de l'amour. Actes du colloque du CORHUM organisé en 2005 par Lucie Joubert, @ nalyses, printemps-été 2006

clairement matriarcal, a son équivalent dans une autre typologie, pour l'auteur tout aussi féminine: le goût de l'élégance, qui va jusqu'au caprice. Plus d'une fois, Rémi Tremblay le chansonnier se fait l'écho de ses collègues masculins pour supplier la femme de son époque de modérer ses ardeurs vestimentaires et, du même coup, diminuer l'ampleur de ses dépenses. Malheureusement, ces demandes conduisent toujours à la même conclusion : les pleurs et les cris. Cela dit, Tremblay donne bien un peu raison à la femme, lorsqu'il s'arrête pour jeter un coup d'œil sur les vêtements, ou plutôt sur les accoutrements, de l'homme :

Un habit qui montre la corde, Sur un pantalon ébréché, Semble crier miséricorde!

Lorsqu'il couvre un homme éméché.

À force de lever le coude, On perce ceux du paletot, Et la femme, qui toujours boude, S'exaspère et dira bientôt :

«Porte donc... porte donc... des trous

Ma foi, ça l'embête, Tu veux toujours te mettre en fête Porte donc des trous (bis) [...]. » («La métaphysique », 1888b, p. 218-219)

Dans un même ordre d'idées, Rémi Tremblay se plaît à dénoncer les arrivistes matrimoniaux, ces hommes qui cherchent à faire un bon mariage avec des femmes mieux nanties qu'eux, financièrement bien sûr, dans le seul but de pouvoir acquérir leurs biens. Ainsi, les hommes n'ont pas toujours le beau rôle; le plus souvent niais, mais quelquefois aussi manipulateurs, particulièrement ceux qui proviennent de la classe bourgeoise, ils sont également dépeints, dans le répertoire de Tremblay, sous des traits qui témoignent d'une réalité peu évoquée dans le XIXe siècle littéraire. Par exemple, l'auteur n’hésite pas à remarquer la propension à la bouteille de plusieurs d'entre eux, et dénonce ouvertement la violence conjugale, qu'il sait présente dans les chaumières; mais là, plus aucun sourire sous cette désacralisation. 
Jean LEVASSEUR, «La poésie humoristique : Rémi Tremblay et les relations amoureuses au XIX ${ }^{\mathrm{e}}$ siècle canadien ", dans La comédie de l'amour. Actes du colloque du CORHUM organisé en 2005 par Lucie Joubert, @ nalyses, printemps-été 2006

\section{Un genre original}

Avec humour et amour, Rémi Tremblay a ainsi témoigné des relations quelquefois difficiles entre les hommes et les femmes, jouant avec candeur sur des réalités autrement occultées par la littérature dite « érudite ». En cela, il demeure un témoin important et privilégié du dernier quart du XIXe siècle canadien-français. S’il avait vécu dans la seconde moitié du XXe siècle, il aurait certainement profité de sa voix de chantre pour enregistrer ses compositions et aurait sans doute alors été comparé à Brassens, Brel, Renaud ou, en Amérique francophone, à La Bolduc, Charlebois, Piché ou Francœur, personnages avec qui il a sans doute plus d'affinités qu'avec les Lamartine, Hugo, Verlaine et consorts, qui formaient à son époque — et aujourd'hui encore - les modèles comparatifs pour la critique canadienne. C'est sans doute ce qui lui permit d'affirmer, au milieu des années 1880, et non sans raison, qu'il avait le sentiment d'avoir créé « un genre à lui » (1886).

\section{Bibliographie}

AnONyme. 1883a, «M. Rémi Tremblay», Le Progrès de l'Est, 10 novembre.

CARrIER, Maurice et Monique Vachon. 1977, Chansons politiques du Québec 1765-1883, Montréal, Leméac, t. I.

CHASsÉ, Paul-P. 1968, Les poètes français de la Nouvelle-Angleterre. 1875 1925, Thèse de doctorat, Sainte-Foy, Université Laval, 472 f.

David, Martine et Anne-Marie Delrieu. 1990, Aux sources des chansons populaires, Paris, Librairie classique Eugène Belin, coll. «Le français retrouvé ».

Doutre, Joseph. 1844, Les Fiancés de 1812, Montréal, RééditionQuébec, 1973. 
Jean LEVASSEUR, «La poésie humoristique : Rémi Tremblay et les relations amoureuses au XIX ${ }^{\mathrm{e}}$ siècle canadien ", dans La comédie de l'amour. Actes du colloque du CORHUM organisé en 2005 par Lucie Joubert, @ nalyses, printemps-été 2006

FRÉCHETTE, Louis. 1888a, «Entre nous », La Justice, 8 novembre, p. 1; reproduit dans L'Indépendant de Fall River, alors dirigé par Tremblay lui-même, le 16 novembre; également paru dans le journal L'Électeur;

—. 1892, Originaux et détraqués, Montréal, Éditions du jour, 1972.

FrÉGAULT, Guy. 1996, Littérature canadienne-française, Montréal, Guérin.

LeVAsseur, Jean, 2004, "Rémi Tremblay (1847-1926); la trépidante histoire d'un journaliste dans les Cantons de l'Est ( $1^{\text {re }}$ partie) », Journal of Eastern Townships Studies / Revue d'études des Cantons de l'Est, $\mathrm{n}^{\circ}$ 24, printemps, p. 31-50;

—. 2004-2005, «Exotisme et historicité : Un Revenant (1884) de Rémi Tremblay », Francographies, no 13 , p. 89-100;

-. 2005a, "Rémi Tremblay», in Bill Marshall (ed.), France and the Americas: Culture, Politics, History, Oxford/Santa Barbara, ABC-Clio, t. III, p. 1158-1159;

—. 2005b, «Rémi Tremblay (1847-1926); la trépidante histoire d'un journaliste dans les Cantons de l'Est ( $2^{\mathrm{e}}$ partie). "Aux chevaliers du nœud coulant" ", Journal of Eastern Townships Studies / Revue d'études des Cantons de l'Est, $\mathrm{n}^{\mathrm{o}} 25$, automne, p. 23-43;

—. 2006, "Aux chevaliers du nœud coulant», dans Pierre Hébert, Yves Lever et Kenneth Landry (dir.), Dictionnaire de la censure au Québec. Littérature et cinéma, Montréal, Fides, p. 57-60.

Mignault, Pierre B. 1883b, «Bulletin bibliographique », La Revue canadienne, vol. 19, nos 11-12, nov.-déc., p. 765.

RoY, Camille. 1923a, Érables en fleurs, Québec, Imprimerie de l'Action sociale, p. 61.

Tremblay, Rémi. 1883c, Caprices poétiques et chansons satiriques, Montréal, A. Filiatreault \& Cie; 
Jean LEVASSEUR, «La poésie humoristique : Rémi Tremblay et les relations amoureuses au XIX ${ }^{\mathrm{e}}$ siècle canadien », dans La comédie de l'amour. Actes du colloque du CORHUM organisé en 2005 par Lucie Joubert, @nalyses, printemps-été 2006

- 1884, Un revenant, édition critique établie, présentée et annotée par Jean Levasseur, Sainte-Foy, Les Éditions de la huit, 2003;

-. 1886, Lettre au major Edmond Mallet, 24 octobre, Université d'Ottawa, Centre de recherche en civilisation canadienne-française, Fonds Suzanne-Lafrenière, P142;

-. 1888b, Coups d'aile et coups de bec, Montréal, Imprimerie GebhardtBerthiaume;

—. 1912, "Si les femmes étaient des hommes», dans Vers lidéal, Ottawa, La Cie d'impr. Commerciale, p. 333;

—. 1923b, Pierre qui roule, Montréal, Librairie Beauchemin. 